\title{
An Improved Non-local Mean Ultrasound Image Denoising Algorithm
}

\author{
Baozhong Liu and Jianbin Liu \\ Computer School ,Beijing Information Science \&Technology University ,Beijing 100101 ,China \\ Software Engineering Research Center, Beijing Information Science \&Technology University ,Beijing 100101 ,China
}

\begin{abstract}
An improved non-local mean ultrasound image denoising method is proposed to solve the problem of poor effect of traditional image denoising algorithm on ultrasound images. In this method the non-local mean filtering algorithm is improved by using the gamma distribution noise model and image block matching, so as to get better noise reduction effect. By comparing with Gaussian filtering algorithm, median filtering algorithm and bilateral filtering algorithm, the improved algorithm achieves better noise reduction than traditional methods, and compared with early non-local mean algorithm in peak signal-to-noise ratio and mean square error performance were optimized.
\end{abstract}

Keywords—non-local means; ultrasound image; despeckle; gamma distribution noise model

\section{INTRODUCTION}

Image denoising is one of the most important issues in image processing. Its purpose is to remove the noise in the image for further processing of the image, such as image analysis, image reconstruction, and image segmentation [1]. Compared with other common imaging methods, ultrasound imaging has the advantages of low cost, non-destructive, non-invasive, real-time, etc. Therefore, ultrasound images are widely used in the fields of industry, medicine and so on. Due to the influence of some factors in the ultrasonic imaging process, ultrasound images are inevitably interfered by noise during the generation process, which causes some important structural details and edge details to be lost in the image. How to retain the edge information and structure information of an image while maintaining effective denoising is a problem in image denoising research. Gaussian filtering algorithm [2], bilateral filtering algorithm [3], anisotropic diffusion algorithm [4], and median filtering algorithm [5] are commonly used noise reduction methods. The denoising effect is achieved to a certain extent but there are also disadvantages that the image structure information cannot be preserved well. Compared with other algorithms, this algorithm has the advantages of stable denoising effect, not blurring details and can better maintain the structural information of edges and images.

The basic idea of the non-local mean filtering algorithm [6] is that the ultrasound image contains many redundant information and there are many very similar image blocks. However, these similar image blocks are distributed over the entire image. Their location may be quite different but the grayscale information is very similar. The NLM method fully explores the similarity of the image itself basing on the existence of the above features, calculates the similarity between the similar blocks and the image block where the noise is present and restores the value of the pixel to be restored by weighted averaging. The algorithm has a good denoising effect on Gaussian noise but the removal effect of the speckle noise of ultrasonic images is not good. Aiming at the shortcomings of traditional non-local mean filtering algorithms, this paper proposes a noise reduction method basing on gamma distribution noise modeland image block matching idea to improve NLM algorithm.

\section{TWO-DIMENSIONAL NON-LOCAL MEAN IMAGE DENOISING ALGORITHM}

In 2005, Antoni Buades proposed a non-local mean algorithm. This algorithm successfully avoids the existing noise reduction algorithm as the premise of the noise image assumption and the loss of image structure information in the process of noise reduction, because the algorithm maintains the edge information. And the excellent performance of the image structure information, so the algorithm has been widely applied and has become a research hotspot in the field of image processing.

The principle of the non-local mean algorithm is as follows: As shown in Figure 1, the figure contains three pixels $P, Y 1$, $Y 2$ and their fields, the domain structure of the pixels $P$ and $Y 1$ is very similar, the pixels $P$ and $Y 2$ The domain structure is very similar. If the $P$ point is filtered, the similarity weight $\omega(P, Y 2)$ of the $Y 2$ pixel point to the $P$ point is greater than the similarity weight $\omega(P, Y 1)$ of the $Y 1$ pixel point to the $P$ point and then the entire image is searched. Find out all the pixels that are similar to the pixel $P$ and finally obtain the filtering result of the pixel $P$ according to the similarity-weighted average[7].

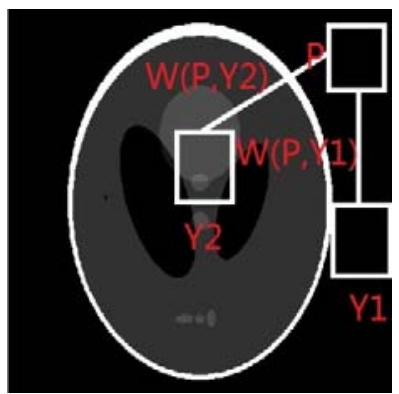

FIGURE I. SCHEMATIC DIAGRAM OF NON-LOCAL MEAN FILTER ALGORITHM

The non-local mean filter algorithm performs image 
denoising by calculating the similarity of pixel points in units of image blocks[8]. A so-called image block is a square field centered on a certain pixel. Set the gray value of the contaminated image at pixel $i$ to $v(i)$ and the filtered gray value to $N L(v)(i)$. For any pixel $i$, the filtered $N L(v)(i)$ can be obtained by computing the weighted average of pixels with similar neighborhoods in the entire noise image:

$$
N L(v)(i)=\sum_{j \in I} \omega(i, j) v(j)
$$

$I$ is the entire image space, and the weight coefficient $\omega(i, j)$ represents the similarity between the pixel $i$ and the pixel $j$. As follows

$$
\omega(i, j)=\frac{1}{C(i)} e^{-\frac{\left\|v\left(N_{i}\right)-v\left(N_{j}\right)\right\|_{2, \alpha}^{2}}{h^{2}}}
$$

$N_{i}$ is a reference block, also called an image block of a pixel to be restored, $N_{j}$ is called a similar block and the two blocks have the same size. $v\left(N_{i}\right)$ represents the gray value of each pixel in the reference block and $v\left(N_{j}\right)$ represents the gray value of each pixel in the similar block. $\left\|v\left(N_{i}\right)-v\left(N_{j}\right)\right\|_{2, \alpha}^{2}$ represents the Gaussian weighted Euclidean distance and $\alpha$ is the standard deviation of the Gaussian kernel. $\omega(i, j)$ satisfies $\omega(i, j) \in[0,1]$ and $\sum_{j \in I} \omega(i, j)=1 . h$ is the attenuation parameter that controls the degree of filtering.

$$
C(i)=\sum_{j \in I} \mathrm{e}^{-\frac{\left\|v\left(N_{i}\right)-v\left(N_{j}\right)\right\|_{2, \alpha}^{2}}{h^{2}}}
$$

is normalized Parameters.

III. IMPROVED TWO-DIMENSIONAL NON-LOCAL MEAN FILTER ALGORITHM BASING ON GAMMA DisTRIBUTION NOISE MODEL AND BLOCK MATCHING

\section{A. Ultrasonic Speckle Noise Model}

The research shows that the early non-local mean filtering algorithm is mainly aimed at Gaussian white noise. The core of weight function calculation is the Gaussian weighted Euclidean distance between the reference block and similar block, so the ideal filtering effect cannot be obtained for the speckle noise. The gamma noise model that is more suitable for the speckle noise characteristics is applied to the non-local average filter algorithm to achieve a better speckle noise filtering effect.

According to the effect of noise on the signal processing process, noise can be divided into multiplicative noise and additive noise. The combination of multiplicative noise and additive noise was proposed by Jain in 1989. The noise model is as follows

$$
u(x)=v(x) \times n_{m}+n_{a}
$$

$v(x)$ is the original image without noise and $u(x)$ is the observed image. $n_{m}$ is multiplicative noise and $n_{a}$ is additive noise. The actual ultrasonic image generation process is complex. All of these factors, such as interpolation operations, envelope detection of RF signals and logarithmic compression operations indicate that Rayleigh and Gaussian models do not have good noise modeling effects on ultrasound images[9]. It is difficult to effectively describe the statistical characteristics of real raw signals using a combination of additive and multiplicative noise. Studies in recent years have shown that the noise of ultrasound images is more consistent with the gamma distribution[10]. The speckle noise model can be changed to

$$
u(x)=v(x)+v^{\beta}(x) \emptyset(x)
$$

The size of the coefficient $\beta$ is affected by the ultrasound imaging equipment. Loupas demonstrated through a large number of experiments on the standard deviation and the mean that the gamma distribution noise of $\beta=0.5$ is more suitable for ultrasound images[11]. In general, we satisfy $\beta=0.5$. $\emptyset(x) \sim N\left(0, \sigma^{2}\right)$ is a Gaussian distribution with a mean of 0 and a variance of $\sigma^{2}$.

\section{B. Improved Non-local Mean Filter Algorithm Basing on Block Matching}

Using image blocks to express the characteristics of pixel points and by similarity between pixel blocks to estimate the similarity between pixel points, we calculate the weight of the mean not by using the similarity between pixel points but Similarity between pixel blocks[12]. Therefore, the structure information of the image will be protected in the denoising process and its performance is better than the conventional filtering method. After the similarity of the two image blocks is obtained according to the traditional Non-local Mean filter algorithm formula, the idea of the above two-dimensional Non-local Mean filter algorithm is to consider the similarity obtained as the similarity between the center points of the two blocks of the reference block and the similar block, that is to say, The similarity obtained by traversing the search area can only be applied to the filtering of one pixel. According to the literature, this paper improves the above algorithm and uses this similarity as the similarity of two image blocks in a noise image. After similarity is calculated, the similarity is multiplied by the gray information of each pixel in a similar block. Degree is used as the recovery result of the similar block to the reference block and it is traversed in the search area according to this method to obtain the result of the product of all the similar blocks and the corresponding similarity and finally the weighted sum is obtained to obtain the value of the reference block to be restored[13].

$$
\omega\left(N_{i}, N_{j}\right)=\frac{1}{C(i)} \mathrm{e}^{-\frac{\left\|v\left(N_{i}\right)-v\left(N_{j}\right)\right\|_{2, \alpha}^{2}}{h^{2}}}
$$

$\omega\left(N_{i}, N_{j}\right)$ represents the degree of similarity between the two-dimensional reference block $N_{i}$, the two-dimensional similarity block $N_{j}$ and still needs to satisfy $\omega\left(N_{i}, N_{j}\right) \in$ $[0,1]$ and $\sum_{j \in I} \omega\left(N_{i}, N_{j}\right)=1$. Under the idea of image block matching, the calculation formula of the $2 \mathrm{D}$ reference block to be restored is

$$
N L(v)\left(N_{i}\right)=\sum_{j \in I} \omega\left(N_{i}, N_{j}\right) v\left(N_{j}\right)
$$


After the above formula is rewritten by the image block matching idea, the workload is saved. However, since the above formula is mainly directed to Gaussian noise, the effect of denoising the ultrasonic image is not ideal. Therefore, an improved Non-local Mean filter algorithm basing on the gamma distribution noise model and image block matching concept will be described below.

For an ultrasound model, satisfying $\beta=0.5$, we assume that

$$
u(x) \mid v(x) \sim N\left(v(x), v(x) \sigma^{2}\right)
$$

Therefore

$$
p(u(x) \mid v(x)) \propto \exp -\frac{(u(x)-v(x))^{2}}{2 v(x) \sigma^{2}}
$$

According to [14], the estimated value of image block $N_{i}$ is rewritten as follows

$$
\hat{v}\left(N_{i}\right)=\frac{\sum_{j=1}^{\left|\Delta_{i}\right|} p\left(u\left(N_{i}\right) \mid v\left(N_{j}\right)\right) v\left(N_{j}\right)}{\sum_{j=1}^{\left|\Delta_{i}\right|} p\left(u\left(N_{i}\right) \mid v\left(N_{j}\right)\right)}
$$

In the formula, $p\left(u\left(N_{i}\right) \mid v\left(N_{j}\right)\right)$ is the probability density function of the image block $u\left(N_{i}\right)$ for the noise-free image block $v\left(N_{j}\right)$ and $\Delta_{i}$ is the search range. Since the image block $v\left(N_{j}\right)$ is indefinite, we use $u\left(N_{j}\right)$ instead of $v\left(N_{j}\right)$, which results in

$$
\hat{v}\left(N_{i}\right)=\frac{\sum_{j=1}^{\left|\Delta_{i}\right|} p\left(u\left(N_{i}\right) \mid u\left(N_{j}\right)\right) u\left(N_{j}\right)}{\sum_{j=1}^{\left|\Delta_{i}\right|} p\left(u\left(N_{i}\right) \mid u\left(N_{j}\right)\right)}
$$

For each reference block $N_{i}$ basing on the gamma noise model, it can be obtained from formula (8) ${ }^{[9]}$ :

$$
\begin{gathered}
p\left(u\left(N_{i}\right), u\left(N_{j}\right)\right) \propto \\
\exp \left(-\sum_{p=1}^{p} \frac{\left(u^{(p)}\left(N_{i}\right)-u^{(p)}\left(N_{j}\right)\right)^{2}}{2 u^{(p)}\left(N_{j}\right)}\right)
\end{gathered}
$$

The similarity distance between two image blocks is calculated as follows

$$
d\left(u\left(N_{i}\right), u\left(N_{j}\right)\right)=\sum_{p=1}^{p} \frac{\left(u^{(p)}\left(N_{i}\right)-u^{(p)}\left(N_{j}\right)\right)^{2}}{u^{(p)}\left(N_{j}\right)}
$$

$p$ is the size of block $N_{i}$. Derivative similarities according to equations (5) and (12) are as follows:

$$
\omega\left(N_{i}, N_{j}\right)=\frac{1}{C(i)} \exp \left(-\frac{1}{h^{2}} \sum_{p=1}^{p} \frac{\left(u^{(p)}\left(N_{i}\right)-u^{(p)}\left(N_{j}\right)\right)^{2}}{u^{(p)}\left(N_{j}\right)}\right)
$$

Similar window size $t$ and search window size $s$ will affect the denoising effect. The value of $t$ has a certain degree of influence on the noise reduction effect. When $t$ is larger, the fine structure information of the image is retained better but the suppression effect of the noise is relatively weak because the algorithm calculates the weight as the window increases. The workload will increase when the value of $t$ decreases, the noise reduction effect of the image will increase but the blur degree of the image will increase relatively. At this time, the calculation amount of the algorithm will be relatively reduced but the image structure information is retained. It will be relatively weak. The selection of the search window size $s$ is very important for balancing noise reduction effects and algorithm efficiency. Although extending the search window within a certain range will increase the peak signal-to-noise ratio, the noise suppression effect will be weakened when the search window is too large and it will also increase. The algorithm takes time to reduce the efficiency of the algorithm.

\section{Image Noise Reduction Evaluation Index}

The effect of image noise reduction is not only judged by subjective vision but also by some commonly used objective evaluation indicators. In this paper, peak signal-to-noise ratio [14](PSNR), mean square error[15](MSE) and structural similarity[16](SSIM) are chosen to evaluate the performance of the algorithm.

a) Peak signal to noise ratio

$$
P S N R=10 \log _{10}\left(\frac{255^{2} \times M \times N}{\sum_{i=1}^{R o w} \sum_{j=1}^{C O l}[f(i, j)-g(i, j)]^{2}}\right)
$$

In the formula, $f(i, j)$ represents the original image, $g(i, j)$ represents the denoised image and $M$ and $N$ represent the rows and columns respectively. The larger value of PSNR, the better filtering effect will be which means the smaller distortion of the filtered image.

\section{b) Mean Square Error}

$$
M S E=\left(\frac{1}{M \times N} \sum_{i=1}^{M} \sum_{j=1}^{N}(f(i, j)-g(i, j))^{2}\right)
$$

In the formula, $\mathrm{f}(i, j)$ represents the original image, $g(i, j)$ represents the denoised image, and $M, N$ represent the rows and columns respectively. The smaller value of MSE, the more similar the original image and the denoised image will be.

c) Structural similarity

$$
\begin{gathered}
S(x, y)=f(l(x, y), c(x, y), s(x, y)) \\
l(x, y)=\frac{2 u_{x} u_{y}+C_{1}}{C_{1}+u_{y}^{2}+u_{x}^{2}}, C_{1}=\left(K_{1} L\right)^{2} \\
c(x, y)=\frac{2 \sigma_{x} \sigma_{y}+C_{2}}{C_{2}+\sigma_{y}^{2}+\sigma_{x}^{2}}, C_{2}=\left(K_{2} L\right)^{2} \\
s(x, y)=\frac{2 \sigma_{x y}+C_{3}}{C_{3}+\sigma_{x} \sigma_{y}}, C_{3}=\frac{C_{2}}{2}
\end{gathered}
$$




$$
\sigma_{x y}=\frac{1}{N-1} \sum_{i=1}^{N}\left(x_{i}-u_{x}\right)\left(y_{i}-u_{y}\right)
$$

In the formula, $x, y$ represent the original image signal and the distorted image signal respectively, $S(x, y)$ denotes the similarity between the distorted signal and the original signal which means the measure of distortion; $l(x, y)$ denotes a two-degree comparison function, $c(x, y)$ denotes a contrast comparison function, $s(x, y)$ represents a structure comparison function and the three functions are relatively independent[17]. $L$ represents the dynamic change of the pixel value and $K_{1}, K_{2} \ll 1, u_{x}, u_{y}$ are the average brightness values as the brightness estimation and $\sigma_{x}, \sigma_{y}$ are the standard variances as the contrast estimation.

\section{COMPARISON AND ANALYSIS OF EXPERIMENTAL RESULTS}

The size of the search area and the similarity window in the non-local mean filtering algorithm has a great influence on the denoising effect. This article has consulted related literature and conducted experiments to determine the size of the selected search area is $7 \times 7$ and the similarity window size is $3 \times 3$. In order to further verify the noise reduction effect of the proposed algorithm, the original images, medical ultrasound images and industrial ultrasound images were processed for noise reduction and to compare with other classical denoising algorithms. So as to objectively evaluate the denoising effect of ultrasound images, the PSNR, MSE and SSIM are selected to evaluate the performance of the algorithm.TABLE1 shows the PSNR, MSE and SSIM of the original image in [18]using different noise reduction algorithms. FIGURE 2 shows the noise reduction effect of the corresponding image. Compared with the algorithm of[18], the proposed algorithm improves the PSNR by $1.0435 \mathrm{~dB}$ and the MSE by 8.5297 . From the denoising effect and data indicators, the PSNR value and MSE value filtered by the algorithm of this paper are all optimal meaning that the degree of distortion of the filtered image is the smallest. From the effect graphs of several filtering algorithms, the images obtained by this algorithm are clearer and the structure and details of the images are better preserved. Considering the algorithm of this paper, the denoising effect is better.

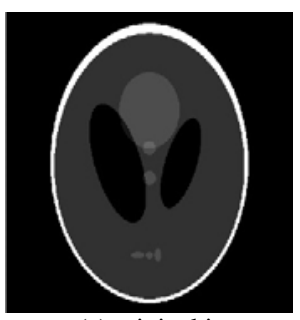

(a) original image

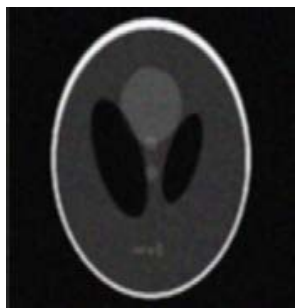

(c) Gaussian

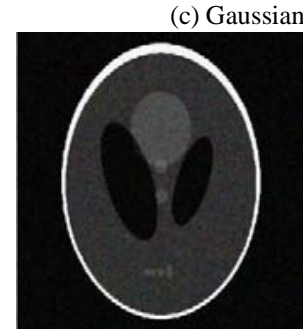

(e) Bilateral

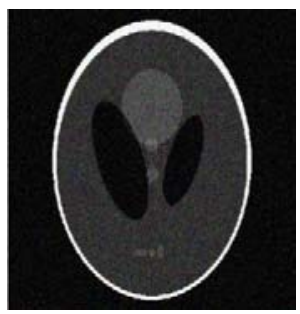

(b) Noise image

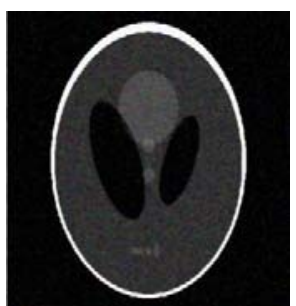

(d) Median

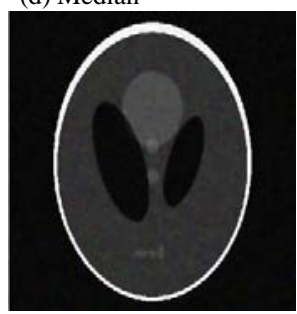

(f) Algorithm
FIGURE II. THE EFFECT OF DIFFERENT METHODS ON IMAGE NOISE REDUCTION

TABLE I. PSNR, MSE, AND SSIM VALUES OF DIFFERENT ALGORITHMS

\begin{tabular}{|c|c|c|c|}
\hline norm & PSNR & MSE & SSIM \\
\hline $\begin{array}{c}\text { Noise } \\
\text { image }\end{array}$ & 22.8384 & 168.4250 & 0.1583 \\
\hline Gaussian & 24.4205 & 111.973 & 0.3959 \\
\hline Median & 31.7933 & 43.0280 & 0.4443 \\
\hline Bilateral & 30.0542 & 64.2181 & 0.2316 \\
\hline NLM & 32.1169 & 39.9379 & $\mathbf{0 . 9 1 7 0}$ \\
\hline Algorithm & $\mathbf{3 3 . 1 6 0 4}$ & $\mathbf{3 1 . 4 0 8 2}$ & 0.4343 \\
\hline
\end{tabular}

Note: The data corresponding to the bold font is the best value for each indicator

\section{CONCLUSION}

After reviewing a large number of documents and analyzing the characteristics of denoising of ultrasonic images, this paper presents a method of denoising ultrasonic images baseing on gamma distribution noise model and image block matching idea to improve NLM algorithm. The original image of[18]was selected for comparison test and compared with several mainstream algorithms. The experimental results show that the proposed algorithm retains good image denoising while retaining better image detail. The data in TABLE 1 shows that compared with the improved weighted NLM algorithm, the peak signal-to-noise ratio is increased by $1.0435 \mathrm{~dB}$ and the mean square error is also reduced. Both of these indexes are optimized but there are deficiencies in the structural similarity. In addition, although the method proposed in this paper has 
achieved a good noise reduction effect and better details are retained but there are still a few details missing so further improvements and research are needed.

\section{ACKNOWLEDGMENT}

The completion of this article is primarily due to the research funding provided by the school. Secondly, I would like to thank the teacher Professor Liwei Zheng and the members of the laboratory for their guidance and help. This paper is awarded the research level of Beijing University of Information Science and Technology by 5211823406.

\section{REFERENCES}

[1] Levin A, Nadler B.Natural image denoising: Optimality and inherent bounds[C]//2011 IEEE Conference on Computer Vision and Pattern Recognition (CVPR). IEEE, 2011: 2833-2840.

[2] Chen P, Qian H, Zhu M. Fast Gaussian particle filtering algorithm[J]. Journal of Huazhong University of Science \& Technology, 2008.

[3] Elad M. On the origin of the bilateral filter and ways to improve it.[J]. Image Processing IEEE Transactions on, 2002, 11(10):1141-1151.

[4] Bai Junqi. Algorithm for Infrared Image Noise Filtering Based on Anisotropic Diffusion[J]. Acta Optica Sinica, 2008, 28(5):866-869.

[5] Huang T, Yang G, Tang G. A fast two-dimensional median filtering algorithm[J]. IEEE Trans.on Acoustic.speech. \& Signal Processing, 1979, 27(1):13-18.

[6] $\mathrm{Xu}$ Yong. Research on image filtering algorithm for edge structure retention type [D]. Hefei University of Technology, 2011.

[7] Yan Nana. Research on degraded image restoration technology based on non-local mean [D]. Yanshan University, 2011.

[8] Zhang Qiong,Li Bin.Formation Principle and Model of Ultrasonic Speckle Noise[J].Electronic Technology and Software Engineering,2014(14):118-120.

[9] Coupé P, Hellier P, Kervrann C, et al. Nonlocal means-based speckle filtering for ultrasound images[J]. IEEE Transactions on Image Processing, 2009, 18(10):2221-2229.

[10] Loupas T, Mcdicken W N, Allan P L. An adaptive weighted median filter for speckle suppression in medical ultrasonic images[J]. IEEE Trans Circuits \& Syst, 1989, 36(1):129-135.

[11] Cai Bin,Liu Wei,Zheng Zhong,Wang Zengfu.An improved non-local mean denoising algorithm[J].Pattern Recognition and Artificial Intelligence,2016,29(01):1-10.

[12] Li L, Hou W, Zhang X, et al. GPU-Based Block-Wise Nonlocal Means Denoising for 3D Ultrasound Images[J]. Computational \& Mathematical Methods in Medicine, 2013, 2013(32):921303.

[13] Kervrann C, Boulanger J, Coupé P. Bayesian Non-local Means Filter, Image Redundancy and Adaptive Dictionaries for Noise Removal[M]// Scale Space and Variational Methods in Computer Vision. Springer Berlin Heidelberg, 2007:520-532.

[14] Hore A, Ziou D. Image Quality Metrics: PSNR vs. SSIM[C]// International Conference on Pattern Recognition. IEEE, 2010:2366-2369.Hore A, Ziou D. Image Quality Metrics: PSNR vs. SSIM[C]// International Conference on Pattern Recognition. IEEE, 2010:2366-2369.

[15] Guo Y, Wang Y, Hou T. Speckle filtering of ultrasonic images using a modified non local-based algorithm[J]. Biomedical Signal Processing \& Control, 2011, 6(2):129-138.

[16] Dong Yubing, Zhang Qishan, Yan Yunping. Image quality evaluation model based on PSNR and SSIM [J]. Journal of Image and Graphics, 2006 (12): 1758-1763.

[17] Zhang Wenjuan,Zhang Lili,Wang Yanhong.SSIM image quality evaluation method based on nonlocal structure tensor[J].Application Research of Computers,2017,34(10):3162-3164+3170.

[18] Zheng Yuanyue,Xu Mingen,Wang Ling.Improved weight non-local mean ultrasonic image denoising[J].Journal of Image and Graphics,2017,22(06):778-786 